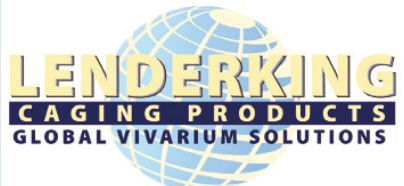

\title{
USDA-compliant vertical socialization for primates
}

Providing vertical space and socialization for nonhuman primates is critical to their psychological well-being. Because primates are arboreal, vertical movement is a natural behavior that should be encouraged, and vertical socialization is a necessary part of primate housing. The US Department of Agriculture (USDA) requires that when nonhuman primates are socialized vertically, each primate's required floor area must be preserved, as specified in the Guide for the Care and Use of Laboratory Animals ${ }^{1}$. Traditional methods of vertical socialization require the removal of a large portion, sometimes all, of the upper cage's floor area. This reduction in floor area will cause one of the primate's floor area to be reduced or eliminated. In these cases, to preserve each primate's required floor area, the footprint of a stacked set of cages would need to be enlarged (possibly doubled), which would consume valuable laboratory floor space. In some cases, removing all or a portion of the upper cage's floor grid exposes personnel to potential injury, due to the size and weight of the grid that must be removed.

\section{Save floor space, protect personnel}

The "Rabbit Hole" Vertical Socialization System (patent pending) developed by Lenderking Caging Products solves the floor space problem and keeps personnel safe. The "Rabbit Hole" concept adds only an inch to a cage rack's footprint. The "Rabbit Hole" is a horizontal rotating panel that controls access between the upper and lower cage compartments by means of a hole in the upper cage's flooring (Fig. 1) and can be easily activated from outside the cage. The easier and safer it is to accomplish vertical socialization, the more often personnel are apt to provide it for laboratory primates.

Here is an example of how the system works. Group IV primates require $6.0 \mathrm{ft}^{2}$ of floor space. In the "Rabbit Hole" system, the floor area of each of the cage compartments is $7.0 \mathrm{ft}^{2}$, increasing the rack's overall footprint by only an inch in width and depth. From outside the cage, personnel can rotate a panel under the upper cage floor to open a $1-\mathrm{ft}^{2}$ hole in the floor. Primates can now socialize vertically, and the required $6.0 \mathrm{ft}^{2}$ of floor area for each primate remains intact.

\section{Provide foraging opportunities}

The "Rabbit Hole" can also be used to create a foraging system by replacing the upper compartment's excreta pan with a clean pan filled with hay and hidden treats and opening the rotating panel. The primate, still with the required minimum floor area, can now forage for treats. The "Rabbit Hole" can be installed in the bottom cage compartment as well, providing an opportunity for the primate in the lower cage to also forage, even when the primates are separated into their own compartments. And, when vertical socialization is in effect, the foraging pan in the lower cage will create more vertical locomotion opportunities as primates travel down to forage and retreat to the upper cage area to eat.

\section{Other benefits}

There are other benefits to the "Rabbit Hole." The upper cage floor and rotating panel can be completely removed from the cage, allowing the whole cage area to be opened, similar to a play cage. This is useful when housing only one primate per rack. And, because there is no lower cage ceiling grid to remove, the panel is lighter and safer to remove. Removing the floor and panel in the wash room makes cleaning and sanitization easier, too.

\section{Company profile}

Founded in 1867, Lenderking is America's oldest caging company. We are committed to providing you with the best solutions and value when housing research animals. We use only American made materials and labor. Our All American ideas have been leading the industry since the last century.

1. Institution for Laboratory Animal Research. Guide for the Care and Use of Laboratory Animals 8th edn. (National Academies Press, Washington, DC, 2011).

This article was submitted to Lab Animal by a commercial organization and has not been peer-reviewed. Lab Animal takes no responsibility for the accuracy or relevancy of the information provided therein.

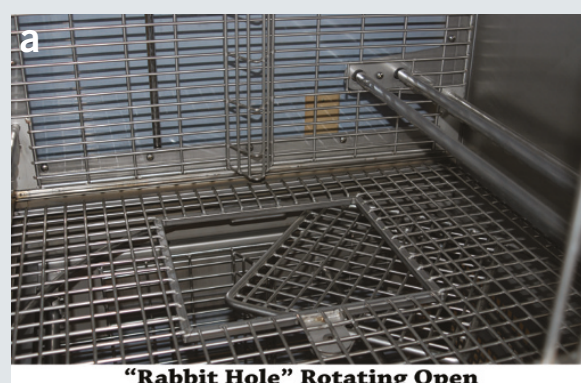

"Rabbit Hole" Rotating Open

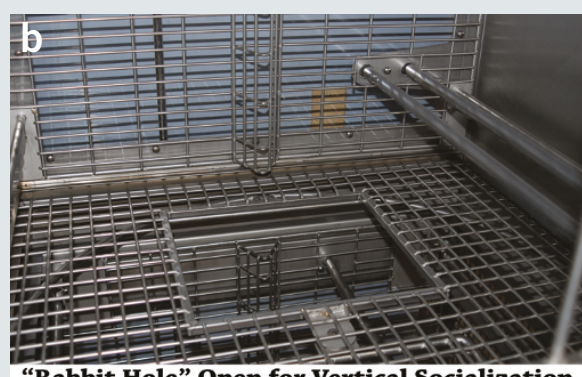

"Rabbit Hole" Open for Vertical Socialization

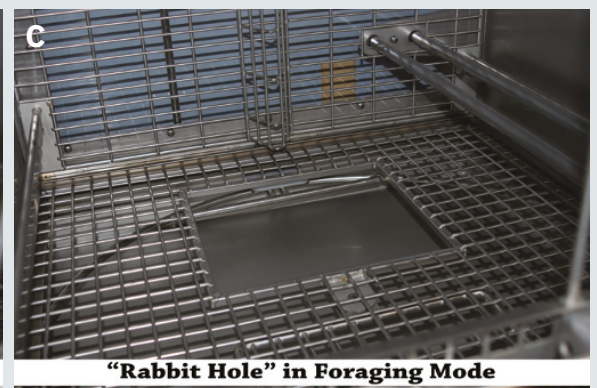

FIGURE 1 | The "Rabbit Hole" vertical socialization system. 